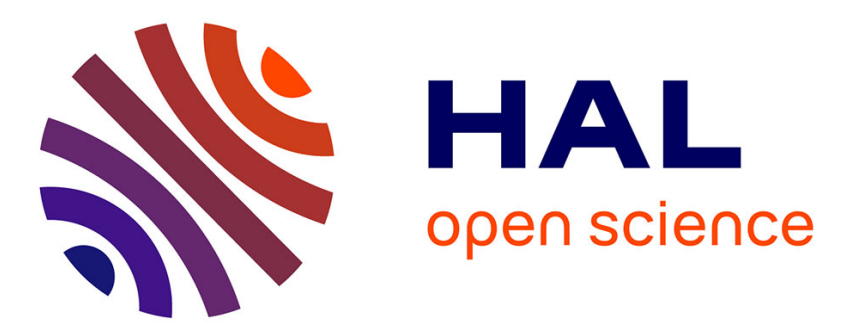

\title{
The mathematical model of the process of the dissolution of the solid particle in the liquid metal under the conducting of the electric current
}

\author{
A. Raichenko, E. Chernikova
}

\section{- To cite this version:}

A. Raichenko, E. Chernikova. The mathematical model of the process of the dissolution of the solid particle in the liquid metal under the conducting of the electric current. Journal de Physique IV Proceedings, 1993, 03 (C7), pp.C7-1229-C7-1233. 10.1051/jp4:19937189 . jpa-00251821

\section{HAL Id: jpa-00251821 https://hal.science/jpa-00251821}

Submitted on 1 Jan 1993

HAL is a multi-disciplinary open access archive for the deposit and dissemination of scientific research documents, whether they are published or not. The documents may come from teaching and research institutions in France or abroad, or from public or private research centers.
L'archive ouverte pluridisciplinaire HAL, est destinée au dépôt et à la diffusion de documents scientifiques de niveau recherche, publiés ou non, émanant des établissements d'enseignement et de recherche français ou étrangers, des laboratoires publics ou privés. 


\title{
The mathematical model of the process of the dissolution of the solid particle in the liquid metal under the conducting of the electric current
}

\author{
A.I. RAICHENKO, A.A. RAICHENKO and E.S. CHERNIKOVA \\ Institute for Problems of Materials Science of Ukrainian Academy of Sciences, 3 Krzhizhanovsky str, Kiev \\ 252180, Ukraine
}

\begin{abstract}
Dissolution process for solid particles surrounded by liquid metal at conducting of electric current essentially differs from dissolution process according to atomic diffusion mechanism. Heterogeneities in liquid conductor at presence of the external physical fields cause a perturbation of this medium's field of velocities. An example of this heterogeneity is solid inclusion. In liquid conductor electric current under influence of some perturbation as a result of interaction with magnetic field raised by this current provokes a motion of fluid which was immovable when an electric current was absent. These motions of conducting fluid will be the more, the more of perturbation of electric current. The spherical solid metal inclusion, immersed in the conducting liquid, represents the given model. The electric current is conducted through the all system. On the basis of magnetohydrodynamics' laws the expression for Stoke's stream function are derived which depended on magnetic permeability and viscosity of liquid, electric conductivity of liquid and solid inclusion, radius of this inclusion and electric current's density. The all variants of the inclusion's conduction were considered: from the until the state.

From the developed theory concludes, that the biggest velocity occurs under lowest (dielectric) and the highest (superconductor) inclusion's conductivity. The liquid flow directions for the cases of the higher and lower inclusion's conductivity in comparison with the liquid's conductivity are opposite. Using the derived formula for stream function stationary diffusion equation is solved, and expression for full diffusional flow from inclusion's surface is obtained. The inclusion's dissolution in the molten metal occurs, providing that the inclusion's components dissolve in the molten metal and diffusion coefficients are not equaled zero. The mechanism of the inclusion's dissolution is the convective diffusion. Dividing the full diffusional flow from spherical inclusion's surface by the full diffusional flow in the case of stationary atomic diffusion, the value named by Sherwood's criterion Sh (or Nusselt's diffusion criterion) may be obtained. The dissolution occurs faster than this process runs by the atomic diffusion with the same temperature.The developed mathematical formalism may be used for analyzing of the processes of the mass transfer in the hard alloys (e.g., WC-Co,Ti-Fe-Ni) and others heterogeneous compositions under electric treatment.
\end{abstract}


Heterogeneities in liquid conduct medium at external physical fields presence cause a perturbation of this medium's field of velocities; no difference we speak about either heterogeneities of hard inclusion's type $(1-3,5,6)$ or a heterogeneity of physical characteristics in area of some space (4). At certain magnetic field's projections to the present speeds' field relatively the perturbations in liquid conductor speeds are stimulated (7). An electric current in a liquid conductor being excited by any manner causes a motion of liquid which was immovable when an electric current was absent. A problem of a flowing of a liquid with a current's presence about an internal spherical inclusion was solved in $(1,2,5-8)$. The inclusion was adopted to be either an insulator or a superconductor. We shell describe analytically speeds of a liquid conductor fluids most generally when an inclusion has an arbitrary conductivity. If we proceed from a standard Navie-Stokes equation, then having summarized a used in work (1) for an nonconduct inclusion method on a case for conduct inclusion, for the condition of absence of a liquid's slip on the inclusion's surface we shall get an expression of the Stokes' stream function.

$$
\psi=-\frac{\mu_{0} \mu_{e} J_{0}^{2} R^{5} \Lambda^{3}}{16 \eta}\left[\left(-\frac{\Lambda^{3}}{2}+1\right)\left(\frac{R}{r}\right)^{2}+\Lambda^{3} \frac{R}{r}-\left(\frac{\Lambda^{3}}{2}+2\right)+\left(\frac{r}{R}\right)^{2}\right] \sin ^{2} \Theta \cos \Theta,(1)
$$

where $\mu_{0}=4 \mathrm{p} 10^{-7} \mathrm{H} / \mathrm{m}$ - is a magnetic constant, $\mu_{\mathrm{e}}$-is a relative magnetic permeability of a liquid medium, $\mathrm{J}_{0}$-is a density of an electric current in liquid on endless distance of the inclusion, $\mathrm{R}$-is a radius of the spherical inclusion, $\eta$-is a liquid's viscosity coefficient, $r$-is a radial coordinate (a center of spherical coordinate system is a center of the inclusion also ), $\Theta$-is an angle coordinate (is measured of the current's direction $\vec{J}_{0}$ ),

$$
\Lambda=\left(2 \frac{\lambda_{e}-\lambda_{i}}{2 \lambda_{e}+\lambda_{i}}\right)^{1 / 3}
$$

$\lambda_{e}, \lambda_{i}$ - are electric conductivities of liquid and the inclusion respectively. Parameter (2) appears in the formula (1) from expression of a current's density in medium with a spherical inclusion (by analogy with formula about electrostatic field (9)).

It's easy to make sure that by changing a conduct inclusion on an nonconduct one, the function (1) is converted to the function received in the work (1). Plots of an equal value of the reducted stream function:

$$
\Psi=\frac{-16 \eta}{\mu_{0} \mu_{e} J_{0}^{2} R^{5}} \psi=-\Lambda^{3}\left[\left(-\frac{\Lambda^{3}}{2}+1\right)\left(\frac{R}{r}\right)^{2}+\Lambda^{3} \frac{R}{r}-\left(\frac{\Lambda^{3}}{2}+2\right)+\left(\frac{r}{R}\right)^{2}\right] \sin ^{2} \Theta \cos \Theta=\text { const } ; \text { (3) }
$$

move further of axis $\Theta=0$ with decreasing of parameter $\Lambda$. The sharpest change of the curve $\Psi=$ const has place when the conductivity of the inclusion equals zero. It means the more lower conductivity of the inclusion, the more higher perturbation has an electric current's field, and the sharper liquid streams turn. Using a formula (1) liquid speed's components turn out from the expression:

$\vec{u}=\overrightarrow{i_{r}} u_{r}+\overrightarrow{i_{\theta}} u_{\theta}=\overrightarrow{i_{r}} \frac{1}{r^{2} \sin \Theta} \frac{\partial \psi}{\partial \Theta}-\overrightarrow{i_{\theta}} \frac{1}{r \sin \Theta} \frac{\partial \psi}{\partial \Theta}$

where $\vec{i}_{r}, \overrightarrow{i_{\theta}}-$ are orts. 
Absolute value of the speed is calculated by a formula:

$u=\sqrt{u_{r}^{2}+u_{\theta}^{2}}$

Shall draw dependencies of the reducted speed:

$U=\frac{16 \eta}{\mu_{0} \mu_{e} J_{0}^{2} R^{5}} u ;(4)$

on the angle $\Theta$ on the lines of constant values of the reducted stream function $\Psi=$ const . Passing from the nonconduct inclusion $(\Lambda=1)$ to more conduct one but keeping a condition $\Lambda>0$ the speeds are removed to a lower. $-2^{\frac{2}{3}} \leq \Lambda<0$ condition's case corresponding to the inclusion with more higher conductivity than liquid's one, passing to the more conductivity (which corresponds to a decrease of $\Lambda$ parameter in algebra sense) causes the speed's increase. As seen in the the speed is close to zero very much at the value of parameter $\Lambda=-0,2$. Electroconvective streams mean to be visible slightly..

Let's turn to an inclusion's substance diffusion to surrounded liquid. Some aspects of this process (for the nonconduct or superconduct inclusion into the liquid conductor) were analyzed in the works $(5,6,8)$. Here a general case will be investigated which is concerned the inclusion of arbitrary conductivity $\Lambda$.

Using Levich's method (10) we may get an equation of a stationary diffusion:

$\frac{\partial c}{\partial \Theta}=\frac{D R^{3} J_{0}}{2} \sqrt{\frac{\mu_{0} \mu_{e} R}{2 \eta} \Lambda^{3}\left(8-\Lambda^{3}\right)} \sin ^{2} \Theta \sqrt{\cos \Theta} \frac{\partial}{\partial \psi}\left(\sqrt{\psi} \frac{\partial c}{\partial \psi}\right) ;(6)$

where $\mathrm{D}$ - is the coefficient of the inclusion substance's diffusion in liquid. A new variable is introduced for solution of the equation (5)

$$
\begin{aligned}
& =\frac{D R^{3} J_{0}}{2}\left[\frac{\mu_{0} \mu_{e} R}{2 \eta} \Lambda^{3}\left(8-\Lambda^{3}\right)\right]^{1 / 2} \int \sin ^{2} \Theta \sqrt{\cos \Theta d \Theta}= \\
= & \frac{4 D R^{2}}{5}\left[\frac{\mu_{0} \mu_{e} R^{3} J_{0}^{2}}{32 \eta} \Lambda^{3}\left(8-\Lambda^{3}\right)\right]^{1 / 2}\left[2 \sqrt{2} E\left(\alpha, \frac{1}{\sqrt{2}}\right)-\sqrt{2} F\left(\alpha, \frac{1}{\sqrt{2}}\right) \sin \Theta \cos ^{3 / 2} \Theta\right]
\end{aligned}
$$

where $F(\alpha, k), E(\alpha, k)$ - are elliptic integrals of 1 and 2 kind respectively,

$\alpha=\arcsin \left(\sqrt{2} \sin \frac{\Theta}{2}\right)$

After an introduction of next variable else with using of (1) and (6)

$z=\frac{\psi^{\frac{1}{2}}}{w^{\frac{1}{3}}}=\left\{\frac{5 R \mu_{0} \mu_{e} J_{0}^{2} \Lambda^{3}\left(8-\Lambda^{3}\right)}{2^{7} \eta D\left[2 \sqrt{2} E\left(\alpha, \frac{1}{\sqrt{2}}\right)-\sqrt{2} F\left(\alpha, \frac{1}{\sqrt{2}}\right)-\sin \Theta \cos ^{\frac{3}{2}} \Theta\right]}\right\}^{\frac{1}{3}}$ 
Solution of the equation (5) by conditions

$c(R)=c_{s},\left.c\right|_{r \rightarrow \infty} \rightarrow c_{0}$

is expressed by formula

$\frac{c_{s}-c}{c_{s}-c_{0}}=\frac{1}{\frac{1}{3} \Gamma\left(\frac{1}{3}\right)} \int_{0}^{z} \exp \left(-\frac{4}{9} z^{3}\right) d z \cdot,(8)$

where $\tilde{\mathrm{A}}(\mathrm{x})$ - is the gamma-function.

Using the determination (8) and considering the expressions of the variable $z$ (7) and parameter $\Lambda$ (2) it's possible to receive a full diffusion flux from inclusion's surface:

$$
\begin{aligned}
& J_{e}=\frac{\left(c_{s}-c_{0}\right) \pi}{1 / 3 \Gamma\left(\frac{1}{3}\right)}\left[\frac{10 R^{7} D^{2} J_{0}^{2} \mu_{0} \mu_{e}}{\eta D} \frac{\left|\lambda_{e}-\lambda_{i}\right|\left(7 \lambda_{e}+5 \lambda_{i}\right)}{\left(2 \lambda_{e}-\lambda_{i}\right)^{2}}\right]^{1 / 3} \\
& \int_{0}^{\frac{\pi}{2}} \frac{\sin ^{2} \Theta \cos ^{1 / 2} \Theta d \Theta}{\left.2 \sqrt{2} E\left(\alpha, \frac{1}{\sqrt{2}}\right)-\sqrt{2} F\left(\alpha, \frac{1}{\sqrt{2}}\right)-\sin \Theta \cos ^{3 / 2} \Theta\right]^{1 / 3}} ;(9)
\end{aligned}
$$

Having divided the full diffusion flux (9) by the full diffusion flux from the surface of the spherical inclusion in the case of a stationary atomic diffusion:

$J_{a}=4 \pi\left(c_{s}-c_{0}\right) R D$

we receive a following expression for a Sherwood's criterion:

$S h=\frac{0.919 \cdot 5^{1 / 3}}{2.32}\left[\frac{R^{4} \mu_{0} \mu_{e} J_{0}^{2}\left|\lambda_{e}-\lambda_{i}\right|\left(7 \lambda_{e}+5 \lambda_{i}\right)}{\eta D\left(2 \lambda_{e}+\lambda_{i}\right)^{2}}\right]^{1 / 3} ;(10)$

Computing for example the value (10) for the case of dissolving of a tungsten's carbide particle into the liquid cobalt binder by conditions: a limiting process is the diffusion of tungsten's atoms, $R \approx 0,5 \mathrm{~mm}$, $\mathrm{T}=1300^{\circ} \mathrm{C}, \mathrm{h} \approx 310^{-3} \mathrm{~kg} / \mathrm{m} \mathrm{c}, \mathrm{J}_{0} \approx 1510^{10} \mathrm{~A} / \mathrm{cm}^{2}, \lambda_{\mathrm{e}} \approx 0.8610^{6} \mathrm{Ohm}^{-1} \mathrm{~m}^{-1}$, $\lambda \mathrm{i} \approx 1.110^{6} \mathrm{Ohm}^{-1} \mathrm{~m}^{-1}(11)$ leads to the value $\mathrm{Sh} \approx 2$.

Present theory may be useful for an analysis of mass-transportation processes in an electrical technology and for elaboration of new technologies which demand using of electro-physical treatments.

References.

[1] Chow C.Y.,Phys. Fluids.(1966), Vol.9,N5,P.933-936.

[2] Chow C.Y., Halat J.A., Phys. Fluids,(1969), Vol.12,N11,P.2317-2322.

[3] Boyarevich V.V., Freyberg Y.G., Shilova E.I., Scherbinin E.V,Riga;Znanie,(1985),P.315.

[4] Alexandrova A.A.,Hizshnyak N.A,Magnetic Hydrodynamics,(1991),N1,P.3-6.

[5] Oreper G.M.,Physical Engineering Journal,(1974), V.27,N6,P.1015-1018.

[6] Oreper G.M, Magnetic Hydrodynamics, (1980),N1,P.72-76.

[7] Oreper G.M, Magnetic Hydrodynamics, (1980),N34,P.69-72. 
[8] Oreper G.M, Magnetic Hydrodynamics,(1979), N3,P.38-42.

[9] Tihonov A.N., Samarskiy A.A,Moscow, GITTL,(1953),P.679.

[10] Levich V.G,Moscow, Physmashgiz,(1953),P.699.

[11] Kikoin L.K, Tables of Physical Values. Reference Book.Moscow,Atomizdat,(1976),8070. 